\title{
Large Area Diamond Disk Growth Experiments and Thermomechanical Investigations for the Broadband Brewster Window in DEMO
}

\author{
Gaetano Aiello, Konstantinos A. Avramidis, Thomas Franke, Gerd Gantenbein, \\ John Jelonnek, Senior Member, IEEE, Andreas Meier, Theo Scherer, Sabine Schreck, Dirk Strauss, \\ Manfred Thumm, Life Fellow, IEEE, Minh Quang Tran, Christoph Wild, and Eckhard Woerner
}

\begin{abstract}
An electron cyclotron system is required in the European DEMO fusion reactor for plasma heating, magnetohydrodynamic control, especially the suppression of neoclassical tearing modes, and thermal instability control. Microwave beams of $2 \mathrm{MW}$, generated by gyrotrons, shall propagate through corrugated $\mathrm{HE}_{11}$ waveguides of $63.5 \mathrm{~mm}$ inner diameter and finally reach specific locations in the plasma by a dedicated set of mirrors. The deployment of the beam may be done either by angular steering, i.e. tilting moveable mirrors, or by frequency steering, i.e. tuning the gyrotron frequency. In the last option, for long pulse operations, broadband optical grade diamond windows are required as confinement and/or vacuum barriers both at the torus and the gyrotron side. The Brewster-angle window represents a promising solution due to its intrinsic simplicity. However, the waveguide aperture imposes a minimum $0180 \mathrm{~mm}$ for the disk at the Brewster-angle of $67.2^{\circ}$ for diamond. This is an ambitious target, considering $140 \mathrm{~mm}$ as the state of the art. First, this paper shows the very promising results of diamond growth experiments carried out by Diamond Materials $\mathrm{GmbH}$ with the first worldwide free-standing optical grade $\varnothing 180 \mathrm{~mm}$ disk having an average unpolished thickness of $1.3 \mathrm{~mm}$. For the first time, one side of this large disk was polished and loss tangent measurements were performed at the center and over a large area. Then, the performance of the window featuring these large disks was characterized by a consistent set of thermo-mechanical analyses in view of the forthcoming window prototyping activity.
\end{abstract}

Index Terms - Brewster-angle window, DEMO, Diamond disk, Electron cyclotron system, Gyrotron output window, Loss tangent, Thermo-mechanical analyses

\section{INTRODUCTION}

$\mathrm{T}$ HE European DEMO reactor shall feature an electron cyclotron system (ECS) able to deploy microwave beams of $2 \mathrm{MW}$ (output gyrotron power) at specific locations in the plasma [1]. This ECS shall be integrated in equatorial ports and two variants are foreseen to develop in the conceptual design

Manuscript submitted April 29, 2021. This work has been carried out within the framework of the EUROfusion Consortium and has received funding from the Euratom research and training programme 2014-2018 and 2019-2020 under grant agreement No. 633053. The views and opinions expressed herein do not necessarily reflect those of the European Commission.

G. Aiello, A. Meier, T. Scherer, S. Schreck, D. Strauss are with Karlsruhe Institute of Technology, Institute for Applied Materials, Karlsruhe, 76131 Germany (e-mail: gaetano.aiello@kit.edu). phase (2021 to 2027) defined by the EUROfusion roadmap [2]. The first option considers port plugs with last mirrors being steered by dedicated actuators for the electron cyclotron (EC) power deposition in the plasma (angular steering). In contrast to the ITER front steering (where moveable mirrors are very close to the plasma) [3], these mirrors are retracted behind the breeding blanket and providing the so-called mid-steering. In this case, the ITER-like chemical vapor deposition (CVD) diamond radio-frequency (RF) window [4] can be used as confinement barrier in the torus and as gyrotron output window with the diamond disk perpendicular to the RF beam direction. However, due to the higher unit power, the window shall be adapted to the waveguide (WG) inner diameter of $63.5 \mathrm{~mm}$ and to the planned two-frequency continuous wave $(\mathrm{CW})$ gyrotron operation at 170 and $204 \mathrm{GHz}$ [5] (1.85 mm thick resonant disk).

The second variant for integration of the ECS relies on frequency step-tunable gyrotrons [6] allowing for a different radial EC power deposition by tuning the gyrotron frequency in the range of few seconds (frequency steering). Target is the tuning in steps of 2-3 GHz over a range of $\pm 10 \mathrm{GHz}$ around the main center frequencies 170 and $204 \mathrm{GHz}$ and even above (e.g., $\sim 238 \mathrm{GHz}$ ) [5]. This option is very attractive because moveable components close to the plasma would be avoided. This would simplify the port plug integration (no actuators and moveable components including their cooling system), maintenance with consequent improvement of reliability and lifetime. A preliminary study on the feasibility to suppress the neoclassical tearing modes (NTMs) by a frequency step-tunable ECS is given in [7].

However, in this case, broadband windows are required and the CVD diamond Brewster-angle RF window concept is under investigation [8] for such high power, $\mathrm{CW}$ tunable gyrotron operation. The feasibility of this window concept for a $50 \mathrm{~mm}$ aperture was successfully shown in a MW-class, D-band,

K. A. Avramidis, G. Gantenbein, J. Jelonnek, M. Thumm are with Karlsruhe Institute of Technology, Institute for Pulsed Power and Microwave Technology, Karlsruhe, 76131 Germany.

T. Franke is with EUROfusion Consortium, Garching, 85748 Germany, and also with Max-Planck-Institute for Plasma Physics, Garching, 85748 Germany.

M. Q. Tran is with Swiss Plasma Center, Lausanne, 1015 Switzerland.

C. Wild and E. Woerner are with Diamond Materials GmbH, Freiburg, 79108 Germany. 
tunable gyrotron prototype, but only for short pulses (a few ms) [9]. Fig. 1 shows the current geometry of the window (details are given in section IV). It can be immediately realized that optical grade (i.e., terminology used by diamond manufacturers to indicate that the diamond is transparent to beam transmission) large area CVD diamond disks are required. A minimum diameter of $180 \mathrm{~mm}$ is necessary for the $63.5 \mathrm{~mm}$ (2.5 inches) aperture (required for $2 \mathrm{MW}$ wave propagation), which is beyond the current technological limits $(\sim 140 \mathrm{~mm})$ of the microwave plasma assisted (MPA) reactors where these disks are usually grown. In order to manufacture larger diamond disks, in the last years, extensive diamond growth experiments have been performed by the industrial partner Diamond Materials GmbH in Freiburg, Germany [10] aiming to the challenging target of a $\varnothing 180 \mathrm{~mm}$ disk with a thickness of about $2.0 \mathrm{~mm}$ (required for an appropriate structural stability and resonant thickness for wrong polarization). The target was met for thermal grade (terminology used by diamond manufacturers to indicate that the diamond can be used only for thermal applications) unpolished disks whereas crack-free optical grade disks were obtained only up to $\sim 0.5 \mathrm{~mm}$ average unpolished thickness [11].

The present paper reports on the successful progress of the optical grade large diamond disk deposition and polishing in section II. In section III, the first loss tangent $(\tan \delta)$ measurements at the disk center and over a large area (mapping) of a $\varnothing 180 \mathrm{~mm}$, optical grade, one side polished disk are presented. Section IV describes the window geometry featuring the large disks including manufacturing aspects which were not considered in previous work [12]. In addition, the analysis work of [12] was further advanced by a new, consistent and parametric set of analyses, described in section $\mathrm{V}$. The main goal was to characterize the performance of this window and to provide the sensitivity of the design itself, an important aspect in the path towards the forthcoming window prototyping activity. Finally, section VI gives an outlook for the activities.

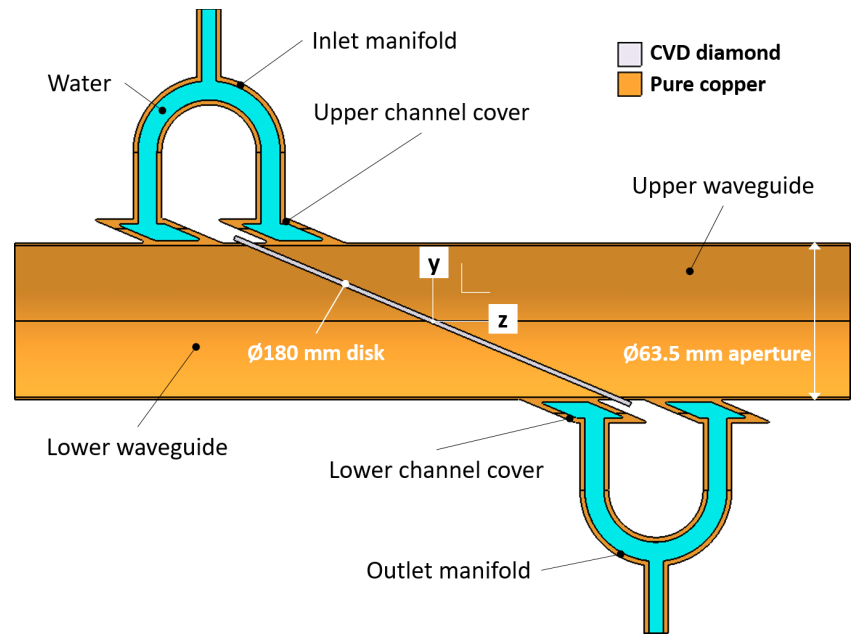

Fig. 1. Section view of the CVD diamond Brewster-angle window for frequency step-tunable $\mathrm{CW}$ gyrotron operation in DEMO. This geometry was used in the analyses of this paper. The names and materials of the involved parts are indicated. The waveguide aperture of $63.5 \mathrm{~mm}$, compatible with an RF power of $2 \mathrm{MW}-\mathrm{CW}$, imposes a minimum diameter of $180 \mathrm{~mm}$ for the disk inclined at the $67.2^{\circ}$ diamond Brewster-angle.

\section{DIAMOND DisK GROWTH EXPERIMENTS}

Current MPA reactors are able to deposit diamond of optical grade only on substrates with diameter 120 to $140 \mathrm{~mm}$, with growth rates in the range of 0.1 to $10 \mu \mathrm{m} / \mathrm{h}$ [13], and polishing procedures are established for disk diameters up to $140 \mathrm{~mm}$. Diamond Materials firstly has pushed the limits of these reactors towards large area deposition with a strong campaign of growth experiments mainly aimed to thermal quality diamond. In fact, the deposition of thermal grade diamond is much faster ( $\sim 10$ times) than the one for optical grade and thus the growth parameters can be better investigated and optimized to fulfill the target. A dedicated stepwise approach has increased the fraction of unbroken thick thermal disks from $65 \%$ in 2018 to more than $90 \%$ in 2019 with the world's first free-standing thermal grade diamond disk having a $180 \mathrm{~mm}$ diameter and average unpolished thickness of about $2 \mathrm{~mm}$ [11]. Diamond Materials has therefore started extrapolating the knowledge on how to grow these disks towards thick optical grade ones.

In 2020, using optimized growth parameters, several optical grade deposition runs with a thickness exceeding $1 \mathrm{~mm}$ were carried out and only $1 / 3$ of the grown disks broke. It is a promising result in the development process towards the target of optical grade $\varnothing 180 \mathrm{~mm} / 2 \mathrm{~mm}$ disks that gives credit to the stepwise approach adopted so far [11]. The main challenge to address in such depositions related to large and thick disks is to avoid generating critical cracks in diamond while assuring a high quality for the RF beam transmission. For this reason, as an example for the optimization process, many efforts have been done by Diamond Materials in re-designing the cooling configuration layout of the MPA reactors in order to decrease at the minimum the stress formation during the diamond growth. Fig. 2a shows the first of its kind free standing, crackfree, optical grade diamond disk of $\varnothing 180 \mathrm{~mm}$ and about $1.3 \mathrm{~mm}$ average unpolished thickness.

In addition, with the increased disk size, a new polishing process has been developed by Diamond Materials and it was used to polish one side (the growth side) of the above mentioned $\varnothing 180 \mathrm{~mm}$ disk until the significant voids were removed. The polished surface of the resulting $\sim 1.2 \mathrm{~mm}$ thick disk shown in Fig. $2 \mathrm{~b}$ is free of significant voids and showed a flatness of better than $6 \mu \mathrm{m}$ over the complete $\varnothing 180 \mathrm{~mm}$ area. However, it shall be noted that it is one thing to polish the disk, but another thing to meet the requirements on planarity and wedge of the disk (to be specified at a later phase). For this purpose, the machine used in this new polishing process has been already designed to adjust the wedge of the disk to a well-defined value while polishing the large disks, but this has not been tested yet. 
a)
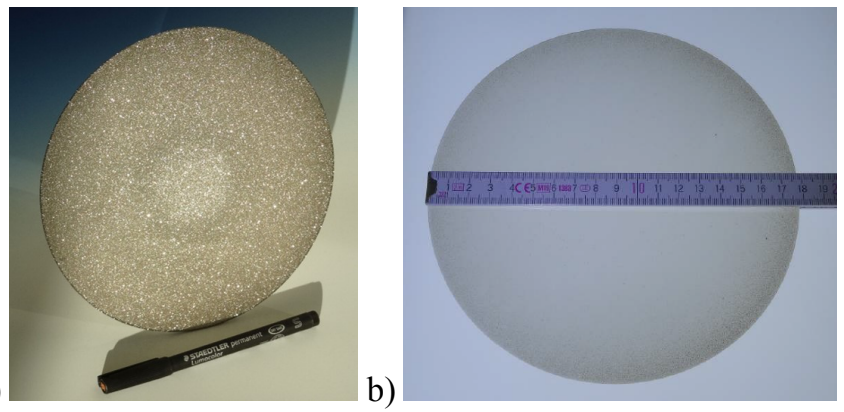

Fig. 2. Worldwide first free-standing optical grade diamond disk with $180 \mathrm{~mm}$ diameter and average unpolished thickness of about $1.3 \mathrm{~mm}$ (a) which, for the very first time, was polished to about $1.2 \mathrm{~mm}$ thickness on one side (the growth side) and no cracks occurred (b).

\section{Loss TANGEnt MEASUREMENTS}

The optical grade diamond depositions at Diamond Materials are usually followed by the $\tan \delta$ measurements [14] at Karlsruhe Institute of Technology (KIT) to provide feedback to the optimization process of the growth parameters. Up to now, it was possible to carry out the measurements only on unpolished portions of cracked disks. They have thus provided only an upper limit for the $\tan \delta$ value $\left(\tan \delta<1 \times 10^{-4}\right.$ in [11]). Looking at Fig. 2b, it was possible to measure the $\tan \delta$ at the center and over a large area ( $\tan \delta$ mapping on $\varnothing 130 \mathrm{~mm}$ ) of the $\varnothing 180 \mathrm{~mm}$, crack-free, optical grade, one side polished diamond disk. The central tan $\delta$ measurement was performed in an open spherical Fabry-Perot resonator and led to the promising value of $1.3 \times 10^{-5}$. As a benchmark, the diamond disks produced by Diamond Materials (of only $\varnothing 70 \mathrm{~mm}$ and $1.11 \mathrm{~mm}$ thickness) for the ITER EC H\&CD system have central tan $\delta$ values lower than $1 \times 10^{-5}$.

An hemi-spherical Fabry-Perot resonator was used for the $\tan \delta$ mapping and the distribution then parameterized in terms of the onset (D10), median (D50) and terminal (D90) parameters, indicating respectively the tan $\delta$ for $10 \%, 50 \%$ and $90 \%$ fraction of the inspected $\varnothing 130 \mathrm{~mm}$ area. However, differently from the standard procedure, due to the thickness variation over the disk (lower than $100 \mu \mathrm{m}$ ), the tan $\delta$ mapping on the polished growth side of the disk required a grid with local thickness values over the inspected area. As shown in Fig. 3, $\tan \delta$ values at $\sim 150 \mathrm{GHz}$ over the large $\varnothing 130 \mathrm{~mm}$ area resulted respectively in $2.7 \times 10^{-5}$ for D50 and $5.6 \times 10^{-5}$ for D90. These values were obtained with an error estimation of $\pm 35 \%$. As a benchmark, in the qualification program of the ITER torus diamond windows, the acceptance criteria defined for D50 and D90 of the tan $\delta$ mapping at $170 \mathrm{GHz}$ in the bare disks are respectively $3.5 \times 10^{-5}$ and $6.0 \times 10^{-5}[14]$. These are again very promising results as it is the first mapping measurement for large area optical grade disks (Ø130 $\mathrm{mm}$ inspected area) whereas the mapping for the ITER torus disks refers to a $\varnothing 60 \mathrm{~mm}$ area only.
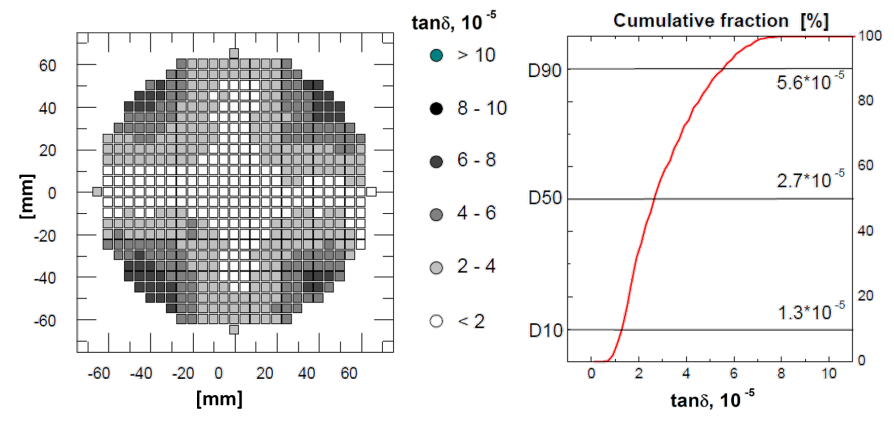

Fig. 3. First-ever tan $\delta$ mapping for $\varnothing 180 \mathrm{~mm}$, polished (growth side only), unbroken, free-standing, optical grade diamond disk (Fig. 2b). The measurement refers to an area of Ø130 $\mathrm{mm}$.

\section{Window GeOMETRY AND MANUfacturing ASPECTS}

Following the conclusions of [9], first basic thermomechanical investigations on the cooling layout of a Brewsterangle diamond window, suited for $50 \mathrm{~mm}$ WG aperture, highlighted the need of cooling channels that must follow the skewed position of the disk [12]. In the work of this paper for the $63.5 \mathrm{~mm}$ WG aperture, beyond considering the larger disk size, the window geometry was also improved taking into account manufacturing aspects in view of the forthcoming window prototyping activity. For instance, looking at the Fig. 1 , the cooling channels are now parallel to the inclined disk (not the case in [12]) and consequently they have a constant cross section along their path, in a parallelogram shape. The window unit has a length of $350 \mathrm{~mm}$ and it features an elliptical diamond disk of $2 \mathrm{~mm}$ thickness, $180 \mathrm{~mm}$ major axis and $95 \mathrm{~mm}$ minor axis. The WGs have an aperture (inner diameter) of $63.5 \mathrm{~mm}$ and wall thickness of $1 \mathrm{~mm}$. Due to the water coolant pressure, the distance between the inner and outer walls in the cooling path is $2 \mathrm{~mm}$. The inner diameter in the inlet/outlet pipes of the manifolds is $6 \mathrm{~mm}$ whereas that in the manifold bodies and in the connecting pipes of the covers is $8 \mathrm{~mm}$. It shall be noted that, the disks, produced in circular shape, shall be then laser cut to an elliptical shape prior to the integration in the window unit.

Fig. 4 shows the assembling sequence proposed for the window prototype. The copper WGs with embedded channels are first welded by electron beam (EB) to the covers to close the channels (box \#a and \#b) and then the resulting parts are brazed to the large diamond disk (box \#c). Most likely, the brazing material will be a copper-silver-titanium (CuAgTi) alloy. Titanium creates a good connection with the diamond disk surface as it has a strong affinity with carbon atoms. Finally, the inlet and outlet manifolds are welded by orbital tungsten inert gas (TIG) forming the joints which close the cooling path (box \#d). The prototype therefore would feature 4 EB (before the brazing), 4 orbital TIG (after the brazing) and 2 brazed joints. The final decision for the assembling sequence of the window prototype is still to be agreed with the involved window manufacturer. 

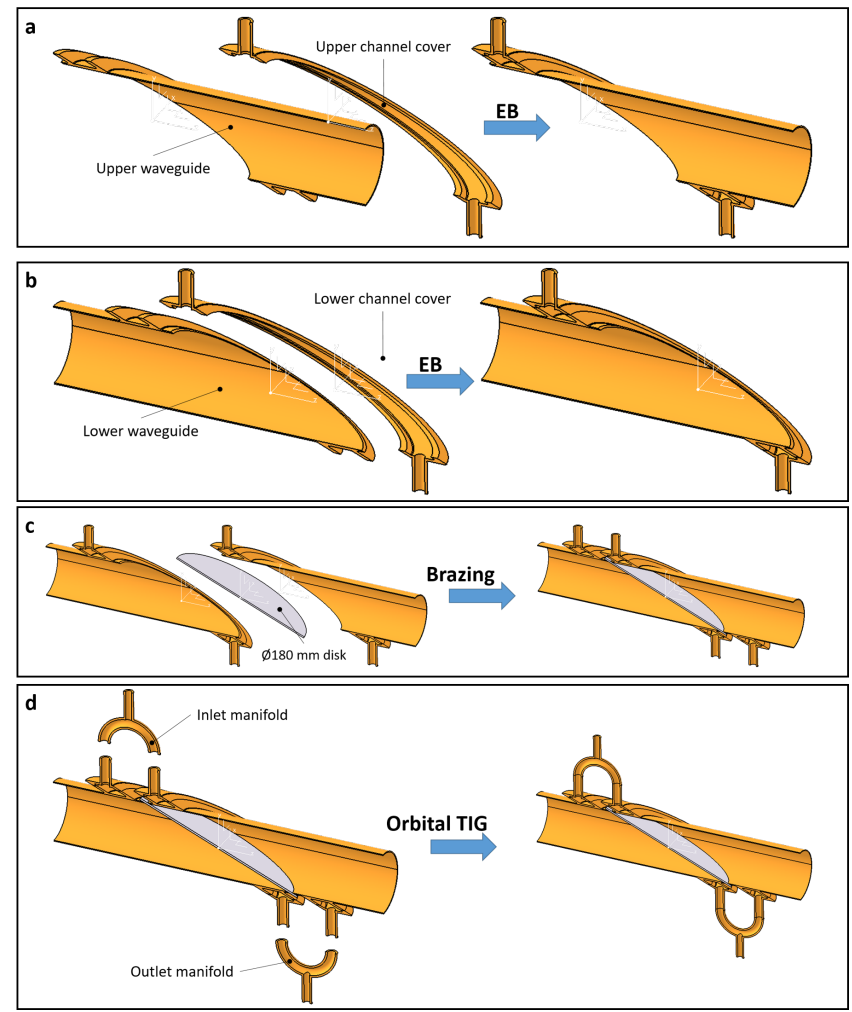

Fig. 4. Assembling sequence (in section view) proposed for the window prototype.

\section{ANALySES}

\section{A. CFD conjugated heat transfer analysis}

Differently from [12], this time a steady-state computational fluid dynamics (CFD) conjugated heat transfer analysis (where fluid and solid domain are both modelled at the same time) was carried out for the geometry of Fig. 1 to investigate the cooling and thermal window performance with respect to a beam power scenario of $2 \mathrm{MW}$ at $204 \mathrm{GHz}$ (reference case). The analysis refers to a gyrotron output window. It should be noted that, due to the transmission losses, the beam power will be lower at the torus window location. For the moment, the transmission losses are considered to be about $15 \%$ from the gyrotrons to the plasma [1]. The code ANSYS CFX V19.2 was used. For symmetry reasons, only half of the window was modelled. Temperature-dependent properties for pure copper and CVD diamond were taken respectively from [15] and [16] whereas the properties directly from CFX's library were used for water. A very fine mesh was generated with $\sim 25.3 \times 10^{6}$ elements. A global mesh size of $1 \mathrm{~mm}$ was applied to the solid model while in the fluid one a global size of $0.3 \mathrm{~mm}$ was adopted and a very much finer mesh (inflation layer with first element size of $10 \mu \mathrm{m}$ ) was generated at the boundary layer to model properly the near wall interactions (heat transfer). For the latter reason, in the analysis settings, the k-omega shear stress transport (SST) model was selected as turbulence model.

A mass flow rate of $0.2 \mathrm{~kg} \mathrm{~s}^{-1}(12 \mathrm{l} / \mathrm{min})$ was assumed for the inlet $\left(0.1 \mathrm{~kg} \mathrm{~s}^{-1}\right.$ in the symmetric model $)$ whereas a reference pressure of zero $\mathrm{Pa}$ was applied to the outlet. The inlet temperature of the water was set to $20^{\circ} \mathrm{C}$. A linearly polarized fundamental Gaussian mm-wave beam was considered. The absorbed power $P_{a b s}$ in the disk was calculated by [17]

$P_{a b s}=\frac{P_{\text {beam }} 2 \pi v \sqrt{\varepsilon_{r}} \tan \delta t}{c_{0} \sin \left(\theta_{B}\right)}$

where $P_{\text {beam }}=2 \mathrm{MW}$ is the beam power, $v=204 \mathrm{GHz}$ is the beam frequency, $\varepsilon_{r}=5.67$ is the permittivity of diamond, $\tan \delta=3.5 \times 10^{-5}$ is the conservative assumed loss tangent (see $\S \mathrm{III}), t=2 \mathrm{~mm}$ is the disk thickness, $c_{0}$ is the speed of light in vacuum and $\Theta_{B}=67.2^{\circ}$ is the Brewster-angle for diamond. The calculation gives an absorbed power of $1546 \mathrm{~W}$ in the Brewster-angle disk. The heat load was then applied to the disk in terms of volumetric power density along the radial coordinate $q^{\prime \prime \prime}(r)$ in $\mathrm{W} \mathrm{m} \mathrm{m}^{-3}$ by

$q^{\prime \prime \prime}(r)=\frac{2 P_{a b s}}{k \pi w_{0}^{2} t} e^{-2 \frac{r^{2}}{w_{0}^{2}}}$

where $k=2.58$ is the correlation factor plane/Brewster-angle disk introduced for the normalization and $w_{0}=15 \mathrm{~mm}$ is the conservative assumed beam waist. The Gaussian distribution was normalized in order to obtain in the Brewster-angle disk the absorbed power of $1546 \mathrm{~W}$.

Fig. 5 shows the temperature distribution in the window. The cooling and thermal performance is good. As expected, the maximum temperature is located at the disk center and amounts to $151{ }^{\circ} \mathrm{C}$, with the coolant at $20^{\circ} \mathrm{C}$ at the inlet. This temperature can be safely accepted, being well below the conservative limit of $250{ }^{\circ} \mathrm{C}$ assumed for diamond. The WGs experience a maximum temperature in the range $58-74{ }^{\circ} \mathrm{C}$ at the central region in contact with the disk. The temperature distribution along the major axis is only slightly non axialsymmetric, in fact the temperature difference between the two edges of the disk along the major axis amounts to $\sim 5^{\circ} \mathrm{C}$ only (see §V.C). Regarding the coolant, the pressure drop in the cooling circuit is 0.47 bar and the temperature increase at the outlet is $1.8^{\circ} \mathrm{C}$ only.

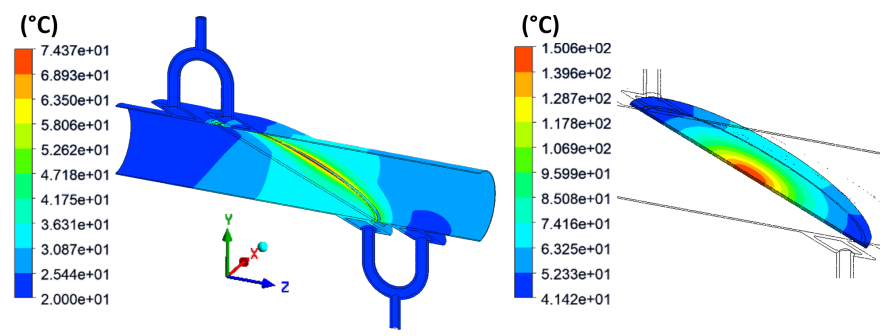

Fig. 5. Temperature distribution in the waveguides (left) and disk (right).

\section{B. Structural analysis for operational phase}

A structural analysis was performed employing the code ANSYS V19.2 to check the stresses generated in the window during operational phase, i.e. with respect to mechanical loads (gravity and coolant pressure) and thermal loads (temperature distribution from $\S \mathrm{V}$.A). As the stresses become close to the yield strength of copper for the temperature range of interest (minimum yield strength of copper for plate products is 
$\sim 62 \mathrm{MPa}$ at $\left.\sim 50^{\circ} \mathrm{C}[15]\right)$, a plastic steady-state structural analysis was therefore carried out with the material temperature dependent properties mentioned in $\S \mathrm{V}$.A. The multilinear isotropic hardening was used as plasticity material model with stress-strain curves given for plate products at different temperatures (up to $250^{\circ} \mathrm{C}$ ). A fixed support was applied to both the ends of the WGs and a reasonable pressure of 10 bar was applied to the cooling interfaces.

Fig. 6 shows the stresses in the window caused by both the mechanical loads and the thermal gradients (temperature differences along the semi-major and semi-minor axes are $\sim 106^{\circ} \mathrm{C}$ and $78^{\circ} \mathrm{C}$ respectively). The WGs experience a maximum stress in the range $\sim 39$ to $59 \mathrm{MPa}$ at the interface region copper-diamond while the stress in the disk is $\sim 45$ to $60 \mathrm{MPa}$ in the outer central region and $\sim 80$ to $105 \mathrm{MPa}$ at the brazing region located in the portion of the disk that forms a very sharp corner with the WGs. In order to obtain more accurate results, the critical stress zone shown in Fig. 7 was further investigated by the submodeling technique in the structural analysis allowing for a much finer mesh in the region of interest. As it can be observed, the stresses in the WGs are slightly higher, in the range 43 to $63 \mathrm{MPa}$. These are entirely caused by the thermal loads (secondary stresses) and can be safely accepted as the minimum ultimate tensile strength of pure copper is $180 \mathrm{MPa}$ at $50^{\circ} \mathrm{C}$ [15]. Also slightly higher stresses were obtained in the disk, being in the range $\sim 88$ to $116 \mathrm{MPa}(\sim 38$ to $50 \mathrm{MPa}$ is the range due to the mechanical loads only), but they are however well below a conservative limit of $150 \mathrm{MPa}$ assumed for diamond (ultimate bending strength is $450 \mathrm{MPa}$ [18]).
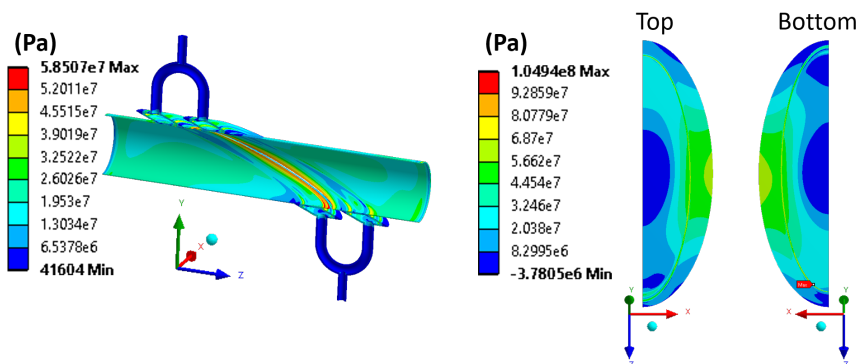

Fig. 6. Distribution of equivalent von Mises stress in the waveguides (left) and first principal stress in the disk (right) due to mechanical and thermal loads.

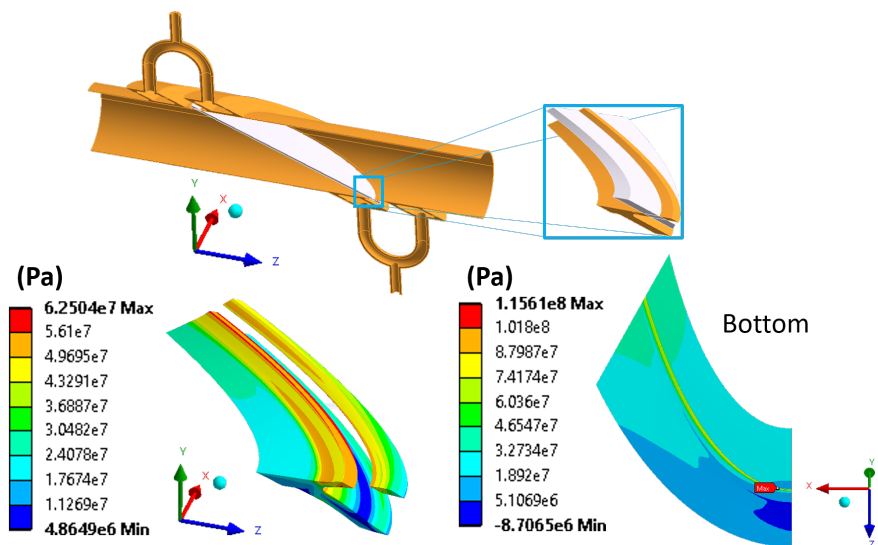

Fig. 7. Submodeling technique applied to the square marked portion of the window: distribution of equivalent von Mises stress in the waveguides portion (left) and first principal stress in the disk portion (right).

\section{Sensitivity study for mass flow rate and beam power}

In view of the upcoming prototyping and later testing activity of the window with large area diamond disks, the sensitivity of the window design with respect to the inlet mass flow rate was first investigated. The analyses in $\S \mathrm{V}$.A and $\S \mathrm{V} . \mathrm{B}$ were repeated for flow rates of $0.33 \mathrm{~kg} \mathrm{~s}^{-1}(201 / \mathrm{min})$ and $0.1 \mathrm{~kg} \mathrm{~s}^{-1}(6$ $1 / \mathrm{min}$ ) and the results are summarized in Fig. 8 and Table I. When the flow rate decreases by $\sim 3$ times, no big change occurs in the temperature distribution. In fact, despite the great reduction in the flow rate, the maximum temperature at the disk center increases by only $\sim 13 \%$ (from $144{ }^{\circ} \mathrm{C}$ to $163{ }^{\circ} \mathrm{C}$ ) and the slight non axially-symmetry increases only from $3.6^{\circ} \mathrm{C}$ to $8.3^{\circ} \mathrm{C}$. This small sensitivity of the design is due to the safe indirect cooling concept (i.e., no direct contact between disk and water) where the heat removal is ruled by conduction in diamond and copper. The choice for the mass flow rate in operational phase of the window shall be thus ruled by the pressure drop. In fact, the $\sim 3$ times reduction in the flow rate leads to a significant decrease from 1.31 to 0.12 bar. The thermal gradients in the disk increase by $\sim 5-11 \%$ leading to a bit more severe stress state, but the stresses are still much lower than the limits.

Then, the same analyses were re-run to investigate the behavior of the window for a worse beam power scenario, $2 \mathrm{MW}$ at $238 \mathrm{GHz}$, leading to an absorbed power in the disk of $1804 \mathrm{~W}$. The results are also shown in Fig. 8. The window still works well assuring good margins against critical temperatures and stresses. The maximum temperature increases by $18 \%$ (still well below the limit) and the thermal gradients become steeper by $\sim 22-24 \%$. The diamond disk experiences consequently greater stresses in the outer central region where they rise from $\sim 45$ to $60 \mathrm{MPa}$ to $\sim 45$ to $75 \mathrm{MPa}$ whereas, in the portion of the disk forming the very sharp corner with the WGs (the region investigated by submodeling technique), the stresses increase from $\sim 88$ to $116 \mathrm{MPa}$ to $\sim 95$ to $124 \mathrm{MPa}$. However, these stresses are still below the limit of $150 \mathrm{MPa}$ assumed for diamond. On the other side, the WGs do not experience any significant change in the stress distribution.

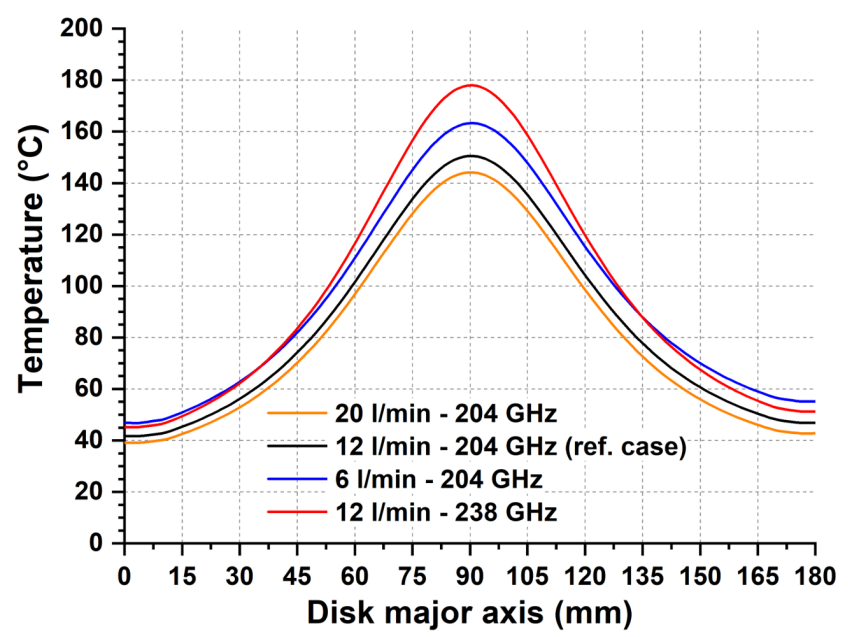

Fig. 8. Temperature profiles along the major axis of the disk for three different values of the inlet mass flow rate and two different beam power scenarios. 
TABLE I

RESULTS FROM SENSITIVITY ANALYSIS FOR INLET MASS FLOW RATE

\begin{tabular}{ccccc}
\hline \hline $\begin{array}{c}\text { Mass flow } \\
\text { rate } \\
(\mathrm{l} / \mathrm{min})\end{array}$ & $\begin{array}{c}\text { Pressure } \\
\text { drop } \\
\text { (bar) }\end{array}$ & $\begin{array}{c}\text { Max T in } \\
\text { disk } \\
\left({ }^{\circ} \mathrm{C}\right)\end{array}$ & $\begin{array}{c}\text { Max T } \\
\text { in WGs } \\
\left({ }^{\circ} \mathrm{C}\right)\end{array}$ & $\begin{array}{c}\Delta \mathrm{T} \text { disk edges } \\
\text { on major axis } \\
\left({ }^{\circ} \mathrm{C}\right)\end{array}$ \\
\hline 20 & 1.31 & 144 & 68.9 & 3.6 \\
12 & 0.47 & 151 & 74.4 & 5.2 \\
6 & 0.12 & 163 & 85.0 & 8.3 \\
\hline \hline
\end{tabular}

\section{Comparative analyses for the brazing disk-waveguides}

The most challenging joint in the window is the brazing process of the disk to the WGs, which is carried out at about 800 to $900^{\circ} \mathrm{C}$ in a vacuum oven and then the temperature of the brazed parts is decreased down to room temperature. The very different thermal expansion coefficients of diamond and copper lead to high stresses at the interface disk-WGs during the cool down phase. In view of the upcoming window prototyping, a last set of structural analyses was thus carried out to investigate this cool-down phase for the window featuring the $\varnothing 180 \mathrm{~mm}$ diamond disks. Such type of analyses have already successfully accompanied the brazing of the Brewster-angle window in [9], but having $50 \mathrm{~mm}$ aperture and no cooling channels. Different constraint configurations shall be experimentally investigated with dummy ceramic disks to reduce the stresses at the minimum while keeping the parts aligned. The analyses can only give an indication to the window manufacturer, especially because properties for copper (stress-strain curves in the range 20 to $800^{\circ} \mathrm{C}$ ) can only be taken from an old reference [19] (however still fine for comparative study at the current stage of the DEMO project). It turned out that the constraint configuration with fixed support at the bottom and sliding support at the top of the window (Fig. 9) is the most promising one. The window is still free to move with the same resulting stress distribution in the disk as in the case of no constraint at the top (upper part completely free to move), but alignment between the upper and lower WGs is kept and the change of the window angle reduces by $\sim 20 \%$ (tilting of the diamond disk). The analyses have also shown that the cooling channels lead to a worse stress state in the disk because they basically act as constraints reducing the possibility for the WGs to move.
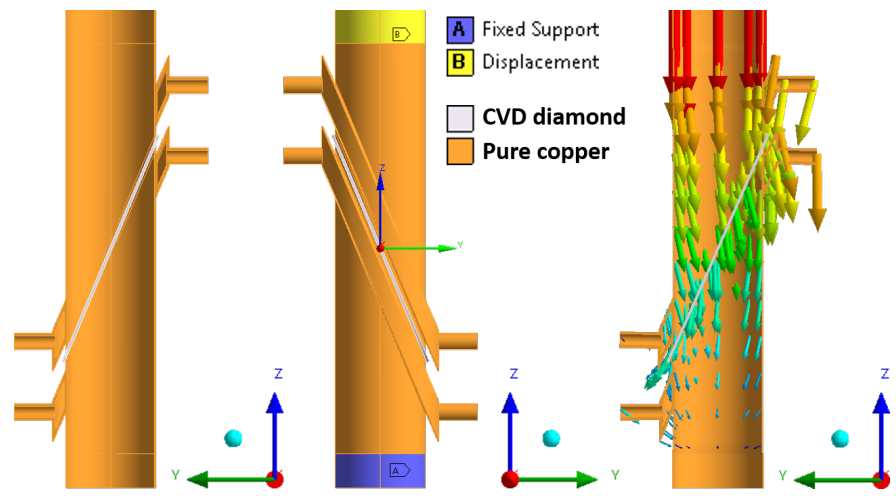

Fig. 9. Geometry (left), boundary conditions (middle) and consequent deformation mechanism (right) for the most promising constraint configuration found in the brazing analysis. The displacement condition refers to a zerodisplacement in $\mathrm{x}$ and $\mathrm{y}$ direction, leaving the upper waveguide free to move vertically.

\section{CONCLUSION}

Within the DEMO conceptual design phase (2021-2027), in close collaboration with industry, diamond growth experiments shall continue to optimize the parameters targeting crack-free optical grade $\varnothing 180 \mathrm{~mm}$ and $2 \mathrm{~mm}$ thick diamond disks. As well, polishing experiments shall continue to achieve a better planarity and wedge while polishing both sides of the $Ø 180 \mathrm{~mm}$ disk, with consequently smaller variation of the thickness over the disk area. Finally, based on the manufacturing strategy and the analyses results discussed in this paper, prototyping and testing activities shall be implemented to show the manufacturing feasibility of the diamond Brewster-angle window featuring large disks and cooling channels for long pulse gyrotron tunable operation in MW-class.

\section{ACKNOWLEDGMENT}

The authors are thankful to Diamond Materials GmbH for the very fruitful collaboration.

\section{REFERENCES}

[1] T. Franke et al., "Integration concept of an Electron Cyclotron System in DEMO," Fusion Eng. Design, vol. 168, 112653, 2021.

[2] T. Donné et al., European research roadmap to the realization of fusion energy, https://www.euro-fusion.org/index.php?id=61\&L=532.

[3] D. Strauss et al., "Nearing final design of the ITER EC H\&CD upper launcher," Fusion Eng. Design, vol. 146, pp. 23-26, 2019.

[4] G. Aiello et al., "FEM analyses of the ITER EC H\&CD torus diamond window unit towards the prototyping activity," Fusion Eng. Design, vol. 161, 112052, 2020.

[5] K.A. Avramidis et al., "Overview of recent gyrotron R\&D towards DEMO within EUROfusion work package heating and current drive," Nucl. Fusion, vol. 59, 066014, 2019.

[6] A. Samartsev et al., "Efficient frequency step-tunable megawatt-class Dband gyrotron," IEEE Trans. Electron Devices, vol. 62, no. 7, pp. 23272332, July 2015

[7] C. Wu et al., "Basic design considerations for a frequency step-tunable electron cyclotron wave system to suppress NTMs in DEMO," Fusion Eng. Design, 2021, to be published.

[8] G. Aiello et al., "Diamond window technology for electron cyclotron heating and current drive: state of the art," Fusion Sci. Technol., vol. 75, no. 7, pp. 719-729, 2019.

[9] G. Gantenbein et al., "First operation of a step-frequency tunable 1-MW gyrotron with a diamond Brewster angle output window," IEEE Trans. Electron Devices, vol. 61, no. 6, pp. 1806-1811, June 2014.

[10] Diamond Materials GmbH, Hans-Bunte-Str. 19, 79108 Freiburg, Germany, https://www.diamond-materials.com/en/.

[11] G. Aiello et al., "Towards large area CVD diamond disks for Brewsterangle windows," Fusion Eng. Design, vol. 157, 111818, 2020.

[12] G. Aiello et al., "Cooling concepts for the CVD diamond Brewster-angle window." Presented at 42th IRMMW-THz. [Online]. Available: 10.1109/IRMMW-THz.2017.8067059.

[13] D.C. Harris, Infrared window and dome materials, SPIE, Washington, 1992.

[14] S. Schreck et al., "ITER ECRH upper launcher: test plan for qualification of the diamond torus window prototype III," Fusion Eng. Design, vol. 109111, pp. 1232-1236, 2016

[15] ITER MPH, Properties for pure annealed copper, ITER IDM reference: 29DEDP.

[16] A. Pai, Properties of diamond produced by chemical vapor deposition process for ITER diagnostic window assembly applications, ITER IDM reference: TMT6EY, September 2016. 
[17] X. Yang et al., "Design of an ultra-broadband single-disk output window for a frequency step-tunable $1 \mathrm{MW}$ gyrotron," Fusion Eng. Design, vol. 74, pp. 489-493, 2005.

[18] M. Thumm, "State-of-the-art of high power gyro-devices and free electron masers," J. Infrared Millim. Terahertz Waves, vol. 41, pp. 1-140, 2020.
[19] M. Merola et al., ITER plasma facing component materials database in ANSYS format, ITER Doc. G17 MD 71 96-11-19 W 0.1, 1997. 